\title{
KX-16: 3D Body Scanning Using Low Cost Depth Sensors
}

\author{
David BRUNER \\ $[\mathrm{TC}]^{2}$, Textile/Clothing Technology Corp., Cary (NC), USA
}

\begin{abstract}
$[\mathrm{TC}]^{2}$ announces in March 2012 the availability of its next generation scanner, the KX-16. It is a significant day in the history of 3D body scanning as the $\$ 10,000$ price point is broken for the first time with a full body coverage, full featured, changing-room sized, color commercial body scanning product with worldwide availability. The KX-16 leverages the emergence of low cost 3D scanning sensors, like the Microsoft XBOX Kinect sensor which utilizes technology from Primesense Corporation.
\end{abstract}

Keywords: 3d body scanner, low cost, kinect

\section{D body scanner KX-16}

$[\mathrm{TC}]^{2}$ is the world leader in 3D body scanning with almost $2003 \mathrm{D}$ body scanning sites around the world supported by a network of ten distributors. [TC] ${ }^{2}$ s first body scanner (3T6) was released in 1997 and was $\$ 200,000$ and required 200 square feet. The KX-16 represents a 20X reduction in price and a 10X reduction in size from that first product.

The KX-16 is [TC] 's fifth generation 3D body scanner technology, and not only does it reach a new unheard of price levels, it also has a new levels of functionality. From our years of involvement in 3D body scanning, we have learned that the key to the commercial deployment of $3 \mathrm{D}$ body scanners is based on three factors:

- Low Price

- Small Size, as to fit in changing room spaces

- End Use embedded functionality

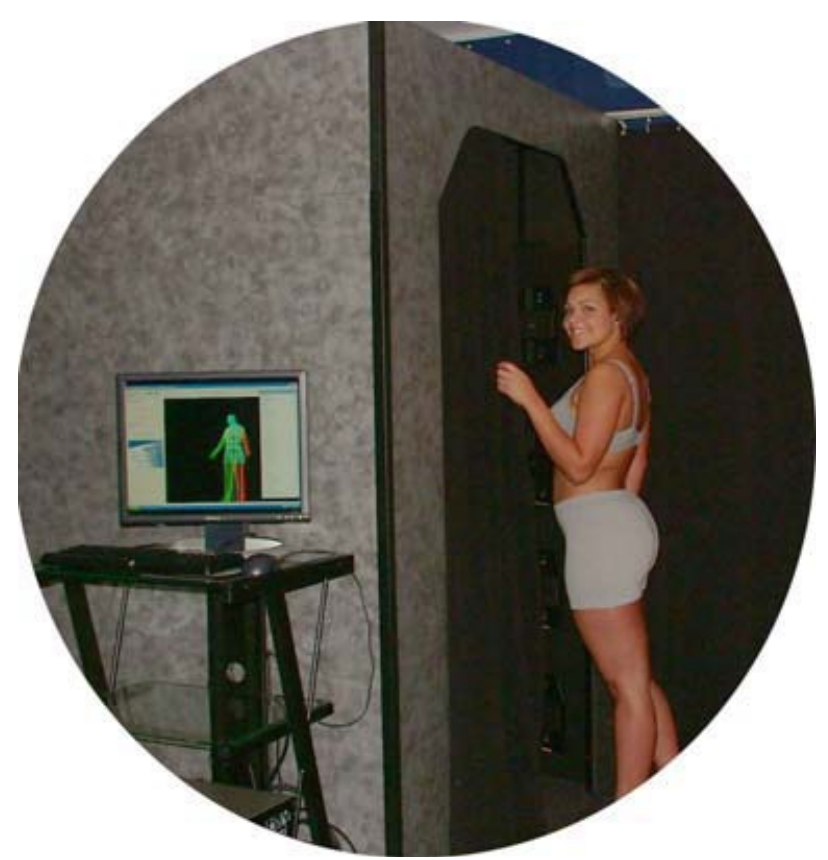

Fig. 1. KX-16 3D Body Scanner. 
The KX-16 answers these market demands. It is one-third the price of [TC] ${ }^{2} \mathrm{~s}$ current market-leading body scanning product, the NX-16, while maintaining its 20 sq.ft. footprint and 16 sensor design. The placement of sensors around the body at four specific heights and at four specific angles was first developed with the NX-16 scanner. This arrangement was determined based on analyzing the body size, shape, and features of tens of thousands of individuals so that the most accurate and complete body scans could be taken of any subject. 3D body scanners have been used for human body surveys over the past twenty years, but the NX-16 and KX-16 scanners are the first scanners designed using data from those same surveys. In addition, having a small footprint is crucial with the new depth sensors as the data quality is excellent close to the body, but is not as good further away.
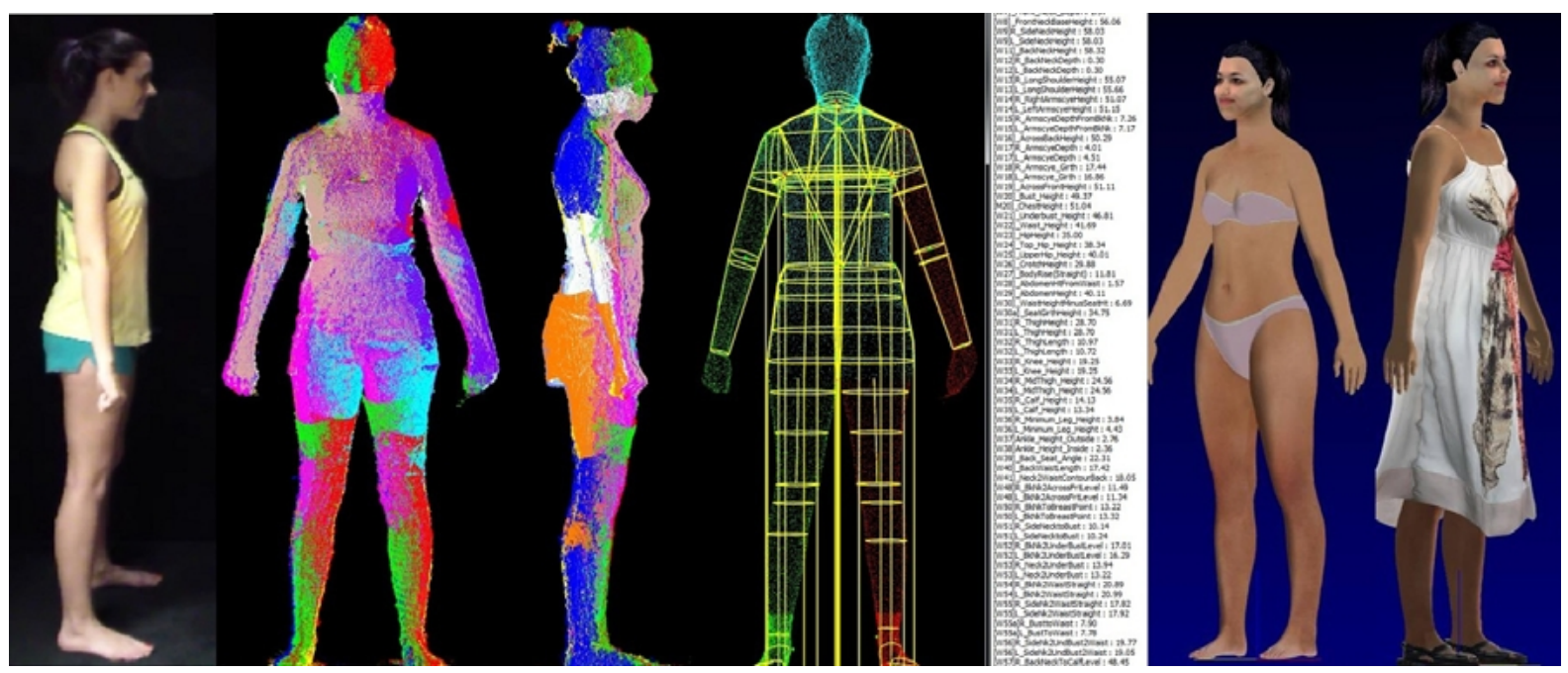

Fig. 2. Scan Subject - Raw Data - Body Model and Measurements - Avatar and Virtual Fashion.

In addition to matching the footprint of the NX-16, the $K X-16$ similarly comes with an attached zero-square footage changing room and the full suite of $[\mathrm{TC}]^{2}$ Body Scanning software functionality including:

- automatic body measurements at over 400 unique points

- manual measurement software features

- embedded size selection for stock size apparel

- embedded Made-to-Measure output features for custom apparel for major apparel CAD systems

- automatic 3D digital avatar creation from the scan including face texturing from a photo

- Virtual Fashion feature to allow virtual try-on of clothes in only "seconds" per garment

- body shape and body composition (body fat) analysis tools

- fully private scan mode with computer voice instructions and self-scan control button

$[T C]^{2}$ 3D body scanner main markets are in the Apparel, Health and Fitness, and Medical application areas and the KX-16 now makes possible many of the business models that both entrepreneurs and majors companies have dreamed about and considered.

The KX-16 matches previous scanner features, and exceeds them in several areas including:

- Color scan data is acquired

- It has low sensitivity to room light or sharp color contrasts on the scan subjects

- Yields over 2 million data points at full body resolution

References

1. An electronic brochure for the KX-16 can be downloaded at http://www.tc2.com/pdf/kx16.pdf 